\title{
Unterstützung gekoppelter hydraulischer Modelle durch Fernerkundungsdaten
}

Zusammenfassung: Die vorliegende Arbeit beschreibt die hydraulischen Verhältnisse der Donau-Auenlandschaft Lobau im Osten Wiens. Die hydraulische Modellierung der Oberflächengewässer und des Grundwassers berücksichtigt die komplexen instationären Strömungsvorgänge in der Lobau, sowie die auentypische Interaktion der beiden Wasserkörper. Fernerkundungsdaten unterstützen die Modellierung in mehrfacher Hinsicht: durch das digitale Geländemodell auf Basis von Laserscanning, das als Grundlage für die Oberflächengewässermodellierung dient; durch Luftbildauswertungen zur Vervollständigung der Vegetationskartierung, die zur Abschätzung der hydrodynamischen Rauhigkeiten dient; sowie durch einen Vergleich der simulierten Wasserflächen mit Luftbildauswertungen zur Überprüfung beider hydraulischen Modelle. Die Simulationen zeigen, dass die Rückströmung der Donau bei einem Durchfluss von beispielsweise $5000 \mathrm{~m}^{3} / \mathrm{s}$ bis zum Groß Enzersdorfer Arm reicht. Die Modellierung erlaubt es auch zu bestimmen, welche Altarmteile durch Grundwasser bzw. durch Oberflächengewässer von der Donau dotiert sind.

Coupled hydraulic modelling supported by remote sensing data

Summary: This paper presents the hydraulic processes of the Lobau floodplain of the Danube East of Vienna. Hydraulic surface water and groundwater models are used to simulate the complex transient flow patterns that are typical of floodplains including surface water - ground water interactions. Remote sensing data assist in the modelling in various ways: in terms of the digitals terrain model that is based on Lidar remote sensing; in terms of the aerial photo interpretation to assist in the vegetation mapping for estimating hydrodynamic roughnesses; as well as in terms of testing the hydraulic models by comparing simulated water areas with aerial photographs. The simulations indicate that the backwater effects from the Danube at a

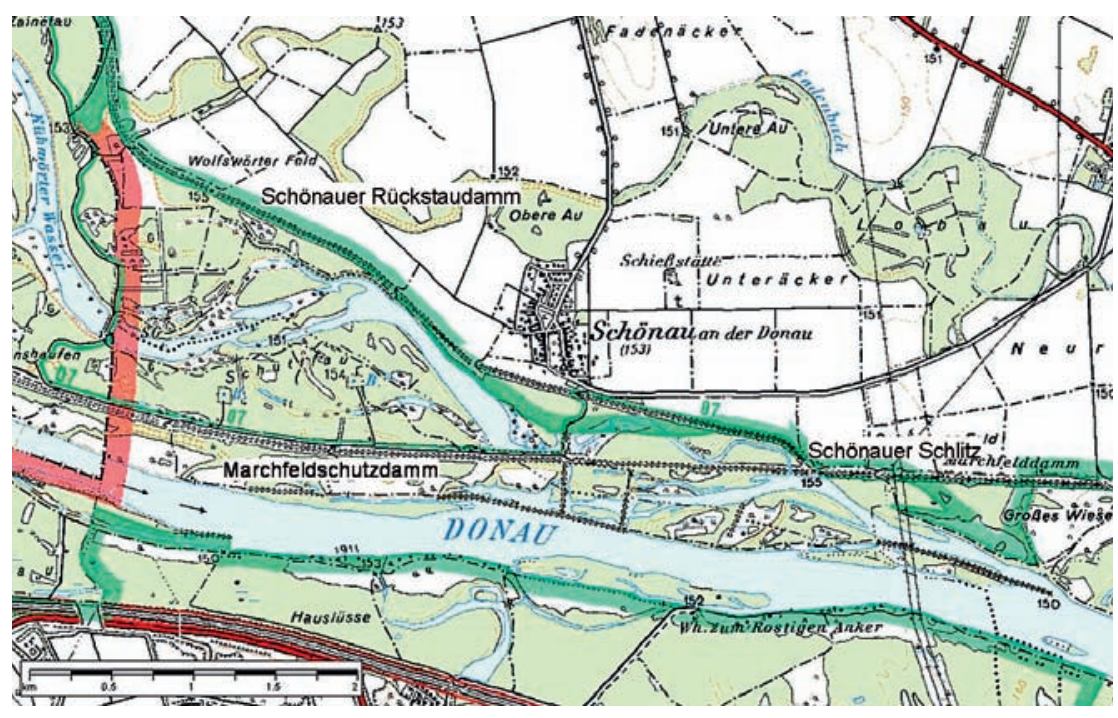

Abb. 1: Lageplan der Lobau , Auszug aus der ÖK 50000.

discharge of $5000 \mathrm{~m}^{3} / \mathrm{s}$ reach as far as to the Groß Enzersdorfer Arm. The modelling also provides information on whether the various parts of the surface water systems are fed by ground water or by surface water from the Danube.

\section{Einleitung}

Das Untersuchungsgebiet ist die Lobau, eine Auenlandschaft im Osten von Wien, die sich am orografisch linken Ufer der Donau von Stromkilometer 1924 bis 1906 erstreckt und ungefähr 2200 ha groß ist. Die Lobau ist ein wertvoller Tier- und Pflanzenraum und zeichnet sich durch einen hohen Artenreichtum aus und steht durch nationale und internationale naturschutzrechtliche Bestimmungen unter besonderem Schutz. Diese Schutzzonen, wie Natura 2000 Gebiet, Biosphärenreservat, RAMSAR-Gebiet, Natur- und Landschaftsschutzgebiet und Nationalpark Donau-Auen, beziehen sich je nach inhaltlicher Ausrichtung auf das gesamte Gebiet der Lobau oder auch nur auf Teile der Unteren Lobau. Eine besondere Bedeutung hat die Lobau weiters durch die vorhandenen Trinkwasserentnahmebrunnen der Stadt Wien, welche auch durch Schutz- und Schongebiete wasserrechtlich geschützt sind. Außerdem dient die Lobau als angrenzendes Erholungsgebiet für die Stadt Wien und niederösterreichische Gemeinden.

Diese vielfältigen Ansprüche an das Gebiet der Lobau erfordern ein gesamtheitliches Management, um eine optimale Entwicklung dieses Raumes zu gewährleisten. Dies erweist sich durch die teilweise gegensätzlichen Ansprüche und die komplexen hydrologischen Verhältnisse als besonders schwierig. In einem dreijährigen Forschungsprojekt wurden nunmehr durch die Entwicklung von Modellen und eines Entscheidungshilfeunterstützungssystems Grundlagen dafür geschaffen, einer für alle Bereiche optimalen Nutzung der Lobau einen Schritt näher zu kommen. Diese Entwicklungen wurden im Rahmen eines interdisziplinären Forschungsprojektes „OPTIMA Lobau“ (Hein et al., 2006; Hein et al. 2008), welches im Rahmen des proVision Programms des BMWF zur Nachhaltigkeitsforschung gefördert wurde, durchgeführt.

In dieser Arbeit wird die hydraulische Beschreibung des Naturraumes Lo- 
bau dargestellt und gezeigt, in welcher Weise Fernerkundungsdaten zur Unterstützung der Modellierung beitragen können.

\section{Untersuchungsgebiet}

Die Lobau wird im Süden durch den Marchfeldschutzdamm zur Donau und im Norden durch den Schönauer Rückstaudamm zum Marchfeld begrenzt (Abb. 1). Beide Dämme dienen dem Hochwasserschutz, wobei eine Anbindung der Lobau an die Donau über eine Öffnung im Marchfeldschutzdamm am südöstlichen Ende der Lobau, dem Schönauer Schlitz, besteht. Die Rückströmung der Donau in das Altarmsystem der Unteren Lobau erfolg über das Äußere Kühwörter Wasser etwa ab einer Mittelwasserführung in der Donau. Bei niedrigeren Donau-Wasserständen strömt Wasser aus der Lobau in die Donau. Als eine derzeitige Managementmaßnahme wird die Lobau über das Mühlwasser aus der Neuen Donau mit maximal $15001 / s$ dotiert. Neben dem Kühwörter Wasser als Hauptgerinne, existieren eine Vielzahl von Nebengewässern, welche ebenfalls teilweise durch einströmendes Donauwasser, aber auch teilweise durch Grundwasser dotiert werden. Der Grundwasserkörper ist Teil des Grundwassergebietes Marchfeld. Den Hauptgrundwasserleiter im Untersuchungsgebiet bilden die rezenten Ablagerungen der Donau die Donauschotter. Die vorhandenen Aufschlüsse zeigen aufgrund des Reliefs des Grundwasserstauers Mächtigkeiten von wenigen Metern bis zu wenigen Zehnermetern. Teile des Untersuchungsgebiets Lobau sind durch Ausand und Aulehm überdeckt, welche stellenweise auch Mächtigkeiten von mehreren Dezimetern aufweisen können.

Die Entnahme von Trinkwasser für die Wiener Bevölkerung erfolgt über fünf Horizontalfilterbrunnen mit einem Gesamtkonsens von 9951/s.

Teile der Lobau werden auch landwirtschaftlich genutzt, wobei diese Nutzung sehr eingeschränkt ist und aufgrund des derzeitigen Nationalparkmanagementplanes in den kommenden Jahren ganz aufgelassen werden soll.

\section{Fernerkundungsdaten für die hydraulischen Modellierungen}

Das Gelände der Auenlandschaft ist flach, weist aber einzelne Senken und Mulden auf, die bei höherer Wasserführung an das Gewässersystem der Altarme angeschlos-

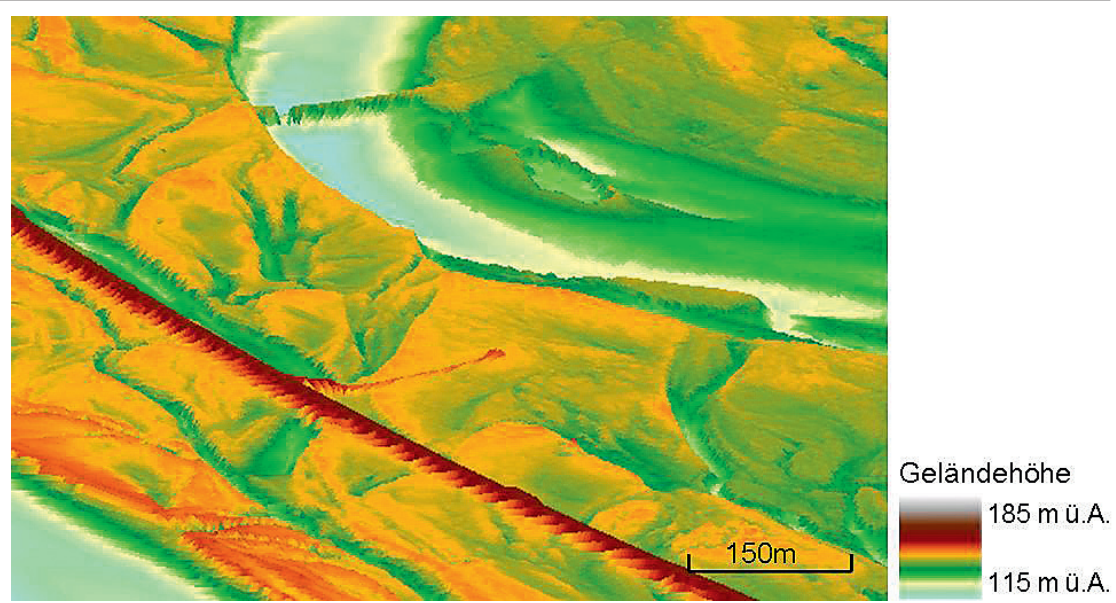

Abb. 2: Detailausschnitt aus dem digitalen Geländemodell der Lobau, Geländehöhen sind 5-fach überhöht dargestellt.

sen sind oder direkt durch Grundwasser dotiert werden. Diese Bereiche sind jedoch für ökologische Betrachtungen von besonderem Interesse, wodurch detaillierter Geländeinformation als Grundlage für die hydraulischen Modellierungen der Oberflächengewässer und des Grundwassers eine große Bedeutung zukommt. Eine Realisierung der genauen Beschreibung der Geländeoberfläche lässt sich durch ein digitales Geländemodell (DGM) erreichen. Das DGM basiert zum Großteil auf Laserscan Daten, welche durch Orthofotos und terrestrische Vermessungen, z. B. an der Gerinnesohle des Altarmsystems, ergänzt werden. Die derzeit übliche Auflösung solcher Laserscans beträgt $1 \mathrm{~m}^{2}$ (Mandlburger et al., 2009), wobei für die Ermittlung der mittleren Geländehöhe unterschiedlich viele Zwischenpunkte Verwendung finden. Für die Ausarbeitung des digitalen Geländemodells, das als Basis der hier vorgestellten numerischen Modelle dient, wurde je nach vorhandener Datengrundlage ein Raster von $2,5 \times 2,5 \mathrm{~m}$ bis $10 \times 10 \mathrm{~m}$ erstellt (Hein et al., 2008).

Das Altarmsystem der Lobau ist außerdem durch Furten und Traversen gekennzeichnet, die im DGM und somit auch im hydraulischen Modell der Oberflächengewässer berücksichtigt wurden.

In Abbildung 2 ist ein Ausschnitt des digitalen Geländemodells dargestellt. Die dunkelbraunen Pixel im Vordergrund der Abbildung zeigen den Hochwasserschutzdamm zwischen Donau und Lobau. Dazu versetzt verläuft der Hauptaltarm (hellblau) der Lobau. Am oberen Rand der Abbildung ist ein Querbauwerk im Altarm zu erkennen, dass die Kreuzgrundtraverse darstellt. Die tatsächliche Öffnung der Traverse ist im DGM als Schlitz berücksichtigt.
Neben den Geländehöhen spielen detaillierte Informationen über die Geländeoberfläche und Landnutzung im Modellgebiet eine große Rolle, da Rauhigkeitsbeiwerte der Gerinne und Überflutungsflächen auf dieser Grundlage basieren. Im Untersuchungsgebiet wurden Biotophaupttypen durch Feldbegehungen kartiert und in Verbindung mit Luftbildauswertungen eine Vegetationskarte (Hein et al. 2008) erstellt. Im Zuge der hydraulischen Modellierung wurde diese Karte in Manning-Strickler Beiwerte übersetzt und als vorab abgeschätzte Eingangsdaten für die Rauhigkeitsverteilung verwendet.

Als komplexe Aufgabe erwies sich die Validierung der hydraulischen Modellierung im Altarmsystem, da nur wenige Wasserstandsmessdaten vorhanden waren. Ein Standbein der Validierung war der Vergleich der simulierten mit den gemessenen Wasserständen. Ein zweites wichtiges Standbein war der Vergleich der simulierten Überflutungsflächen mit Auswertungen der Wasserflächen aus Luftbildern. Diese Methode erlaubte es auch, die Dotation von nicht angeschlossen Gräben und Senken durch Grundwasser zu überprüfen. Der Vergleich mit den Fernerkundungsdaten ist somit eine zusätzliche Verifizierung der Ergebnisse der Grundwassermodellierung. In diesem Zusammenhang ist auch nochmals auf die große Bedeutung eines genauen Geländehöhenmodells hinzuweisen, welches auch der Erstellung von Flurabstandsplänen diente.

\section{Gekoppelte hydraulische Modellierung}

Die komplexen Strömungsvorgänge in der Lobau sind durch die Rückströmung der 
Donau bei entsprechenden Wasserständen und der damit verbundenen Änderung der Fließrichtungen gekennzeichnet. Die Überflutungsflächen in der Lobau sollen zur ökologischen Bewertung flächendetailliert dargestellt werden. Aus diesen Gründen ist eine zwei-dimensionale Modellierung des Untersuchungsgebietes erforderlich. Die Ergebnisse der hydraulischen Oberflächen- und Grundwassermodellierungen umfassen die Berechnung der Wasserspiegellagen sowie Fließgeschwindigkeiten und Fließrichtungen. Die Interaktion zwischen Oberflächengewässer und Grundwasser ist im Untersuchungsgebiet durch die vorhandenen Gräben und Senken, welche nicht ständig von der Donau dotiert werden, von besonderer Bedeutung.

Diese notwendige Kopplung des Grundwassermodells an das Oberflächengewässermodell erfolgt über die simulierten Wasserspiegelhöhen der Altarme, die als Leakageobjekte im Grundwassermodell berücksichtigt werden. Die einseitige Kopplung der Grundwassermodellierung an die Oberflächengewässermodellierung ist zulässig, da die Interaktion der beiden Wasserkörper weitgehend durch den Einfluss der Oberflächengewässer bestimmt wird.

\subsection{Das Oberflächengewässermodell}

Die Oberflächengewässermodellierung beruht auf dem zwei-dimensionalen numerischen Modell TUFLOW (Syme, 1991; WBM 2006), das auf der Methode der Finiten-Differenzen basiert und die Geländeoberfläche des Modellgebietes durch einen regelmäßigen Raster mit Zellen entsprechender Höhe diskretisiert. Die Hochwasserschutzdämme sind im hydraulischen Modell zusätzlich durch Geländebruchlinien erfasst. Die Grenzen des Modellgebiets werden im Norden durch den Schönauer Rückstaudamm und im Süden durch das orografisch rechte Ufer der Donau definiert. Im Westen reicht das Modellgebiet bis zum Tischwasser in der Oberen Lobau, im Osten bis zum Stromkilometer 1906 der Donau. Das Gesamtraster des Rechenmodells ist $13700 \times 9000 \mathrm{~m}$ und besteht aus 409000 aktiven Elementen. Ein Rasterelement ist 10 x 10 m. Zur Berücksichtigung der Rückströmung in die Lobau umfasst das Rechenmodell nicht nur das Altarmsystem der Lobau, sondern auch die Donau von Stromkilometer 1919,40 bis 1906,20. Der Unterwasserspiegel der Donau bei einem bestimmten Durchfluss definiert eine Randbedingung für das Oberflächengewässermodell. Er wurde auf Basis der Was- serspiegellagenberechnungen der Donau (Fischer-Antze und Gutknecht, 2005) festgelegt. Die Simulationen wurden instationär durchgeführt, wobei unterschiedliche stationäre Wasserführungen der Donau als Randbedingung angenommen wurden. Sie reichten vom sommerlichen $\mathrm{Nie}$ derwasser bei $910 \mathrm{~m}^{3} / \mathrm{s}$ bis zum höchsten Die Zuflussrandbedingung im Mühlwasser der Oberen Lobau bezieht sich auf die Dotation der Oberen Lobau, die gemäß Wasserrechtsbescheid begründet ist.

Die Manning-Strickler Beiwerte des Augebietes basieren auf der bereits erwähnten Vegetationskarte. Sie wurden für die unterschiedlichen Vegetationstypen aus Literaturwerten abgeschätzt und im Zuge der Modellkalibrierung angepasst. Diese Modellkalibrierung erfolgte für einen Durchfluss der Donau Q von $3000 \mathrm{~m}^{3} / \mathrm{s}$.

Die Simulationsergebnisse der Wasserspiegellagen der Donau wurden anhand der numerischen Berechnungen der Wasserspiegellagen der Donau (FischerAntze und Gutknecht, 2005) überprüft. Die Validierung der simulierten Wasserspiegellagen im Altarmsystem erfolgte schiffbaren Wasserstand bei $5000 \mathrm{~m}^{3} / \mathrm{s}$.

durch einen Vergleich der simulierten Überflutungsflächen mit Wasserflächen, die aus Luftbilden bestimmt wurden.

\subsection{Das Grundwassermodell}

Für die vorliegenden Untersuchungen konnte auf ein bereits für das gesamte Marchfeld vorhandene kalibriertes Grundwassermodell zurückgegriffen werden, welches im Rahmen der Umweltverträglichkeitserklärung für das „Flussbauliche Gesamtprojekt der Donau östlich von Wien“ (Blaschke, 2004) erstellt wurde. Die besonders detaillierte Berücksichtigung des Altarmsystems der Lobau benötigte eine Adaptierung diese Modells, welche zu einer Verfeinerung der räumlichen Diskretisierung und Einbeziehung auch kleinerer Altarme in das Grundwassermodel führte. Aufgrund der Möglichkeit der klaren Festlegung der Modellrandbedingungen wurde weiterhin das gesamte Marchfeld als Modellgebiet festgelegt. Die Modellgrenzen bilden im Süden die Donau und im Norden der Weidenbach. Im Westen reicht das Rechenmodell bis zur Abzweigung des Marchfeldkanals von der

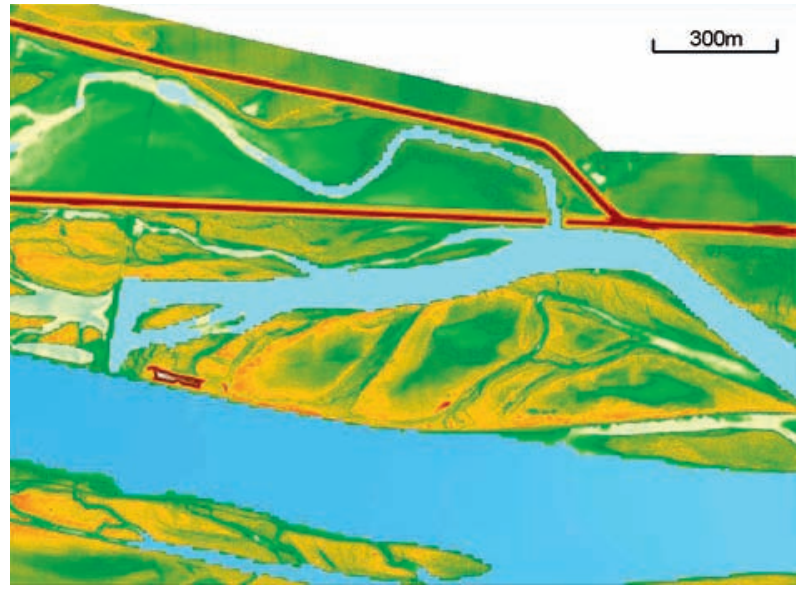

Abb. 3: Untere Lobau mit dem Schönauer Schlitz. Anbindung des Altarmes über das Auß Kühwörter Wasser bei einem stationären Durchfluss der Donau von $2200 \mathrm{~m}^{3} / \mathrm{s}$. Farben wie $2200 \mathrm{~m}^{3} / \mathrm{s}$. Farben wie zeigt größere Wassertiefe.

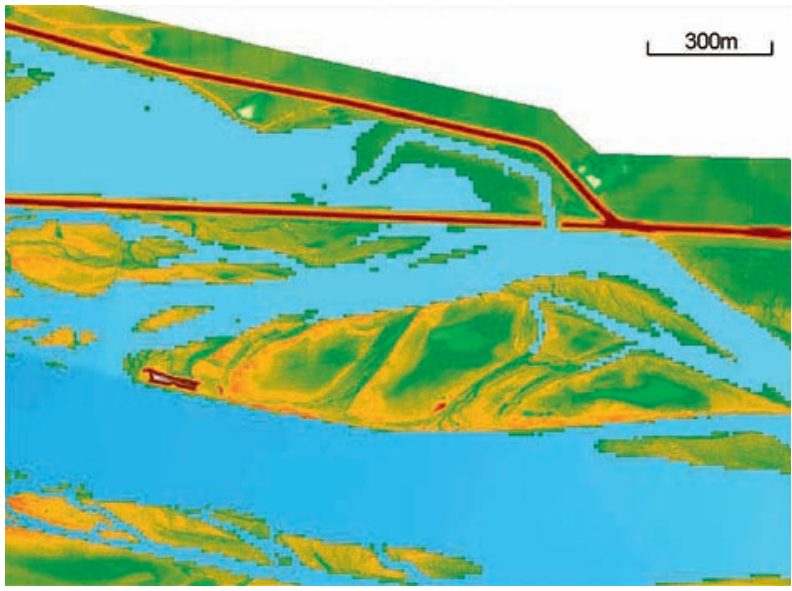

Abb. 4: : Wie Abb. 3 jedoch bei einem stationären Durchfluss der Donau von 4000 m³/s. 


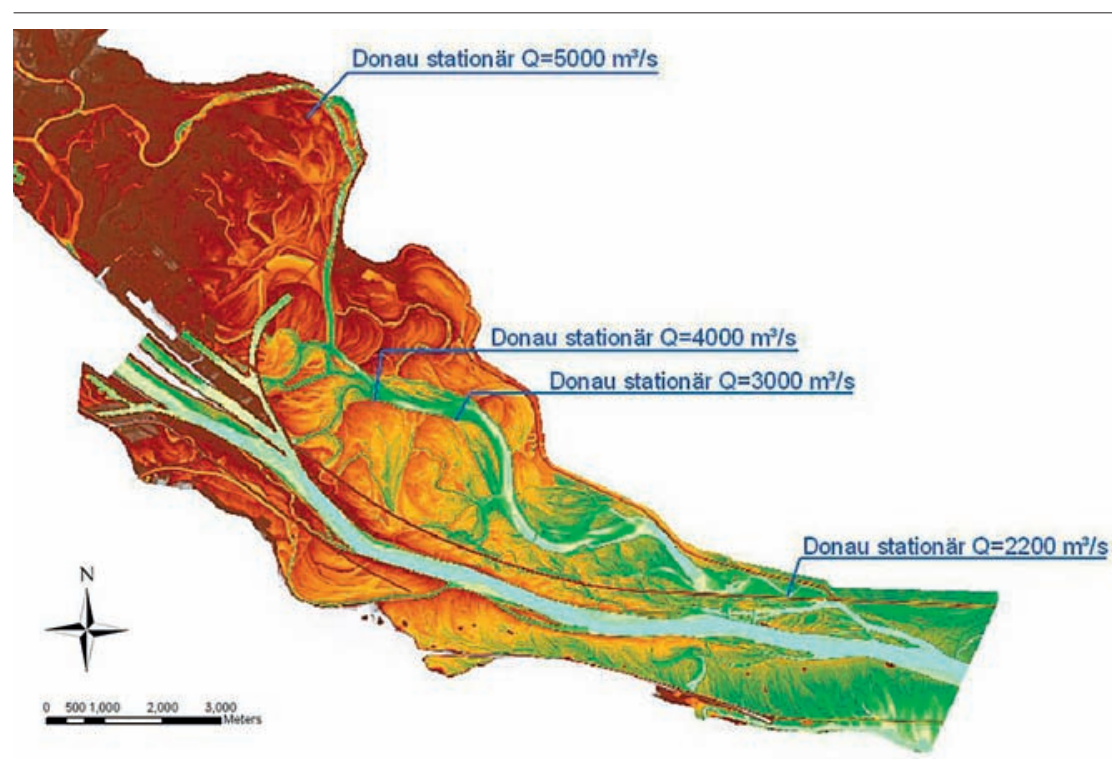

Abb. 5: Simulierte Rückströmungsbereiche der Donau in der Lobau unter verschiedenen stationären Bedingungen in der Donau, Darstellung des digitalen Geländemodells für den Modellbereich. Farben wie Abb. 2.

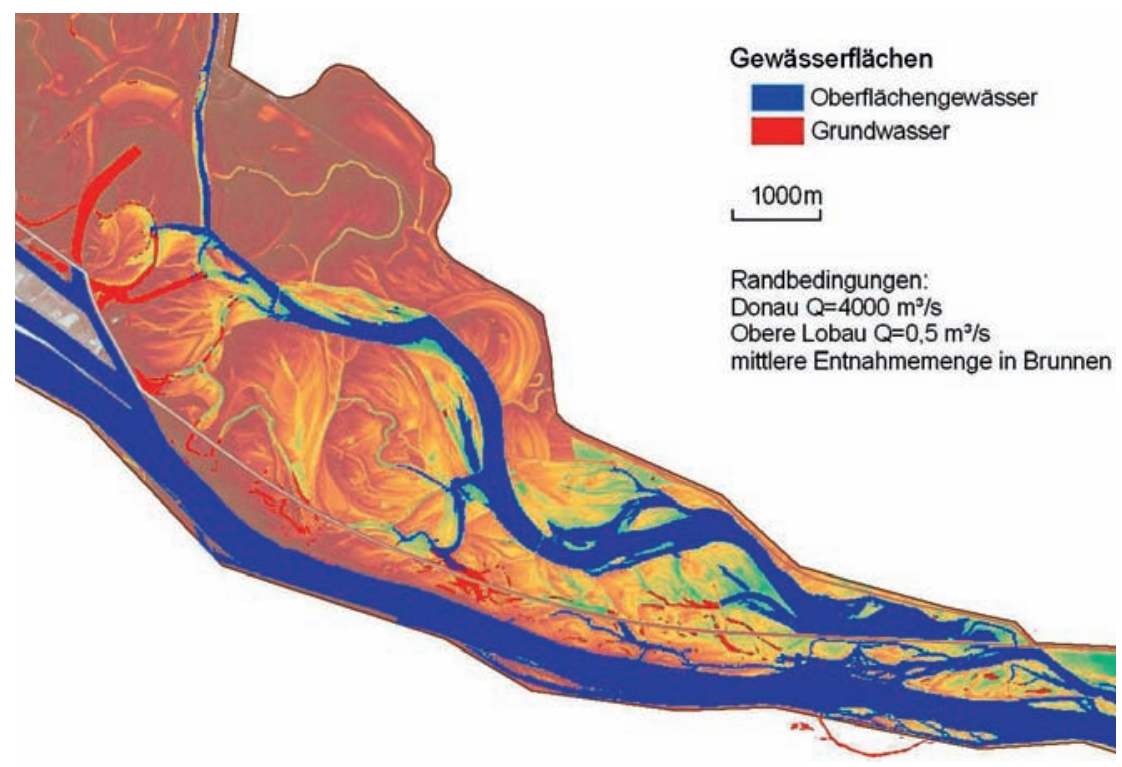

Abb. 6: Lobau. Blau: Gewässerflächen, die aus Oberflächengewässern dotiert sind: Rot: Gewässerflächen, die aus Grundwasser dotiert sind. Durchfluss der Donau von 4000 m³/s. Im Hintergrund digitales Geländemodell.

Donau und im Osten bis zur March. Die verwendete Software HPP_GMS (Blaschke und Blöschl, 1992) beruht auf der Methode der Finiten-Elemente und besteht aus 7600 Knoten und 14930 Elementen.

Für die stationäre Neukalibrierung des adaptierten Modells wurden ein niederer und ein mittlerer Systemzustand herangezogen. Als Kalibrierungsgröße wurde die Transmissivitätsverteilung verwendet. Die Randbedingungen, wie Grundwasserneubildung und Grundwasserzuflüsse, wurden aus dem bestehenden Grundwassermodell übernommen.

\section{Ergebnisse und Diskussion}

Abbildungen 3 und 4 zeigen die simulierten Wasserflächen bei stationären Durchflüssen in der Donau von $2200 \mathrm{~m}^{3} / \mathrm{s}$ und $4000 \mathrm{~m}^{3} / \mathrm{s}$. Die Abbildungen umfassen die Darstellung des Altarmes am östlichen Ende der Unteren Lobau zwischen den zwei Hochwasserschutzdämmen. Die Anbindung der Unteren Lobau an die Donau erfolgt durch eine Öffnung im Marchfeldschutzdamm, dem Schönauer Schlitz, über das Äußere Kühwörter Wasser.

Die in Abbildung 3 dargestellte Situation zeigt, dass die Untere Lobau bei sommerlichem Mittelwasser der Donau (Hohensinner etal.,2008) gerade angeschlossen ist und die Strömung im Hauptgerinne des Altarms Kühwörter Wasser nur den ersten Geländehochpunkt erreicht. Die Wassertiefen betragen in diesem Bereich jedoch nur noch wenige Zentimeter. Abbildung 4 zeigt die quasi-stationären Zustände in der Lobau bei einem Durchfluss in der Donau von $4000 \mathrm{~m}^{3} / \mathrm{s}$. In der Unteren Lobau sind bereits weite Bereiche überströmt und es bilden sich geschlossene Wasserflächen. Auch das Gewässersystem des Äußeren Kühwörter Wassers weist einen deutlich höheren Anbindungsgrad an die Donau auf.

Abbildung 5 zeigt zusammenfassend wie weit sich die Rückströmung der Donau in der Lobau bei unterschiedlichen Donauwasserführungen maximal ausbreitet. Die stationären Randbedingungen der Donau umfassen dazu Simulationen bei $2200 \mathrm{~m}^{3} / \mathrm{s}, \quad 3000 \mathrm{~m}^{3} / \mathrm{s}, \quad 4000 \mathrm{~m}^{3} / \mathrm{s}$ und $5000 \mathrm{~m}^{3} / \mathrm{s}$. Die Darstellung erfolgt anhand des digitalen Geländemodells. Aus den Analysen der Strömungsvektoren geht hervor, dass der Rückströmungsbereich der Donau bei $2200 \mathrm{~m}^{3} / \mathrm{s}$ wie bereits erwähnt im Bereich des Schönauer Schlitzes verbleibt. Bei einem Durchfluss von 3000 $\mathrm{m}^{3} / \mathrm{s}$ erreicht die Rückströmung die Mühlleitner Furt, bei $4000 \mathrm{~m}^{3} / \mathrm{s}$ die Kreuzgrundtraverse und bei $5000 \mathrm{~m}^{3} / \mathrm{s}$ den Großenzersdorfer Arm.

Die flächendetaillierten Flurabstände ergeben sich durch Verschneiden der simulierten Grundwasserspiegellagen mit dem digitalen Geländemodell. Grundwasserflächen, die höher als die Geländeoberfläche liegen, sind in Abbildung 6 für einen Donaudurchfluss von $4000 \mathrm{~m}^{3} / \mathrm{s}$ in rot dargestellt und ergänzen das Gewässersystem der Oberflächengewässer (in blau). Abbildung 7 zeigt einen Detailausschnitt eines Luftbildes im Bereich des Ölhafens Lobau. Die in rot dargestellten Flächen sind vom Grundwas- 


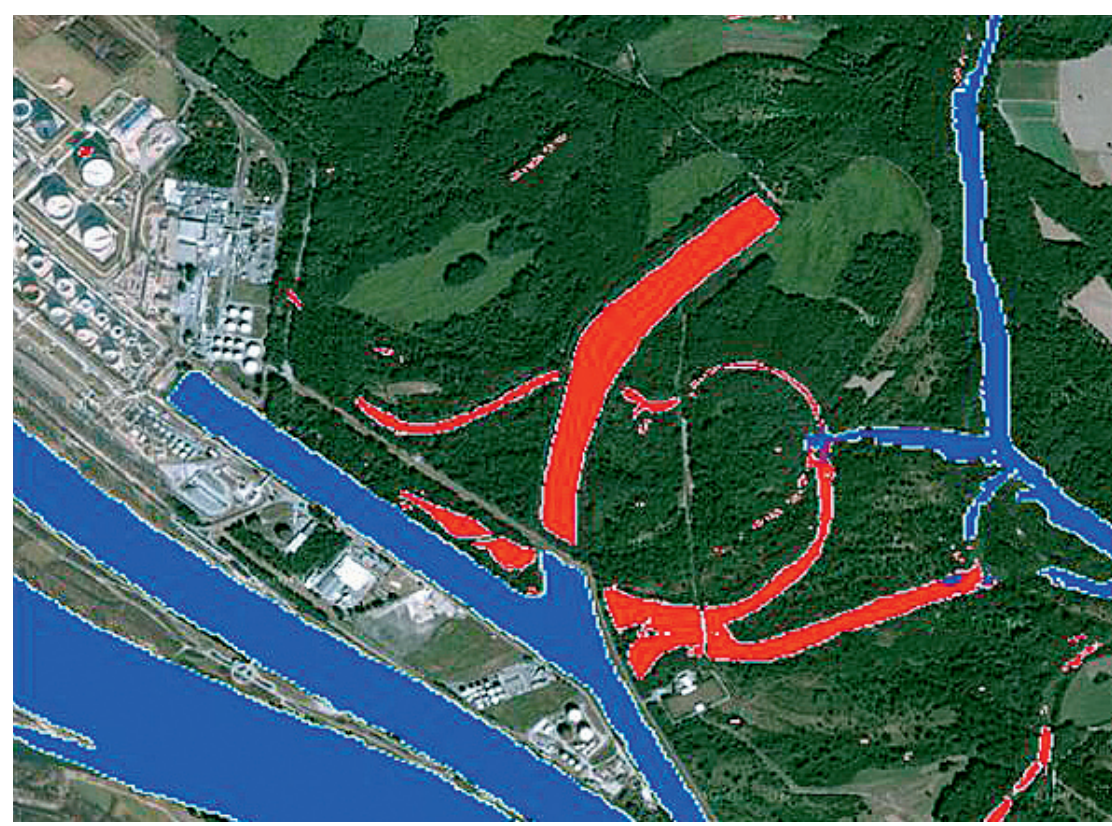

Abb. 7: Grundwasser Dotierung von Lacken und abgeschnittenen Altarmen. Blau: Gewässerflächen, die aus Oberflächengewässern dotiert sind: Rot: Gewässerflächen, die aus Grundwasser dotiert sind.

ser dotierte Gewässerflächen. Es handelt sich dabei um den Donau-Oder Kanal, der nicht an das Gewässersystem angeschlossen ist, sowie um weitere abgeschnittene Altarme. Die in Abbildung 7 dargestellte Überlagerung der Wasserflächen aus der Modellierung mit jenen aus den Luftbildern zeigt eine sehr gute Übereinstimmung, wodurch die gute Kalibrierung der Modelle bestätigt wird. Dies ist insbesondere für die hier geforderten flächenhaften Modellaussagen über Nass- flächen bei unterschiedlichen Donauwasserführungen von großem Wert.

\section{Zusammenfassung}

Die detaillierte Kenntnis der hydraulischen Verhältnisse in der Lobau ist als Grundlage für eine optimale und zwischen den unterschiedlichen Interessensgruppen abgestimmte Nutzung der Lobau notwendig. Die im Rahmen des Forschungsprojektes „OPTIMA Lobau“ er- stellten numerischen Modelle bieten die dafür notwendige Grundlage. Aufgrund der schwierigen Geländesituation und der notwendigen flächendetaillierten Aussage über Nassflächen, kommt dem Einsatz von Fernerkundungsmethoden wie Laserscanning und Luftbilder eine besondere Bedeutung zu. So hat sich bei den Modellerstellungen und den Auswertungen der Modellergebnisse gezeigt, dass ein digitales Geländemodell mit der hohen Genauigkeit des Laserscannings unerlässlich ist. Die Verwendung von Luftbildern für die flächenhafte Erfassung von der Vegetation und für die Modellvalidierung zeigen den großen Nutzen der Fernerkundung bei der hydraulischen Modellierung.

\section{Danksagung}

Die hydraulische Modellierung der Lobau erfolgte im Rahmen von „Optima Lobau“, ein interdisziplinäres Projekt aus dem proVision Programm des BMWF, das sich der Forschung für nachhaltige Entwicklung widmet. Für zusätzliche finanzielle Unterstützung wird dem Lebensministerium, dem BMVIT, den Magistratsabteilungen 22, 31, 45 und 49 der Stadt Wien, dem Land Niederösterreich und dem Nationalpark Donau-Auen gedankt.

\section{Korrespondenz:}

DI Christine Gschöp

TU Wien

Institut für Wasserbau und Ingenieurhydrologie

Karlsplatz 13, 1040 Wien

Tel.: 58801-22322

E-Mail: gschoepf@hydro.tuwien.ac.at

\section{LITERATUR}

Blaschke AP, Blöschl G (1992) "Defining a Pre and Post-processing Environment for Groundwater Modelling". Hydrocomp ,92. International Conference on Interaction of Comdraulics and Hydrology. 583-589. Budapest. Blaschke AP (2002) Auswirkungen der Selbstdichtung auf das Grundwasser in staugeregelten Flüssen. Forum fur Hydrologie und Wasserbewirtschaftung, Heft 1, Fachgemeinschaft Hydrologische Wissenschaften in der ATVDVWK, München, BRD.

Blaschke AP (2004) Grundwasserströmungsmodell für das generelle Projekt - „Flussbauliches Gesamtprojekt - Dona Wien". Bericht Grundwasserströmungsmodell. Auftraggeber via donau.

Fischer-Antze T, Gutknecht D (2005) Durchführung von Wasserspiegellagen an der Donau. Auftraggeber BMVIT. Endbericht, Institut für Wasserbau und Ingenieurhydrologie, Technische Universität Wien.

Hein T, Blaschke AP, Haidvogl G, Hohensinner S, Kucera-Hirzinger V, Preiner S, Reiter K, Schuh B, Weigelhofer G (2006) Optimised management strategies for the Biosphere reria decision support system, Ecohydrology \& Hydrobiology, Vol. 6, No. 1-4, 25-36. Hein T (2008) Umweltverträglichkeitserklärung zum „Flussbauliches Gesamtprojekt - Donau östlich von Wien". Fachbeitrag Wasserwirtschaft-Grundwassernutzung. Auftraggeber via donau.

Hein T, Baart I, Blaschke AP, Habereder C Haidvogl G, Hohensinner S, Preiner S, Reckendorfer W, Reiter K, Schuh B, Stanzer G, Weigelhofer G (2008) Zusammenfassender Bericht. Optimised management of riverine Support System: merging societal requirements and ecological development in a changing world Ortima Lobau. ProVision Project ging world. Optima Lobau. ProVisi Hohensinner S, Herrnegger M, Blaschke AP, Habereder C, Haidvogl G, Hein T, Jungwirth M, Weiß M (2008) Type-specific reference conditions of fluvial landscapes: A search in the past by 3D-reconstruction, Catena 75 8) $200-215$

Kaleris V (1986) Erfassung des Austausches von Oberflächen- und Grundwasser in horizontalebenen Grundwassermodellen. Institu für Wasserbau, Universität Stuttgart, Heft 62. Mandlburger G, Höfle B, Briese Ch, RessI C Otepka J, Hollaus M, Pfeifer N (2009) Topographische Daten aus Laserscanning als Grundlage für Hydrologie und Wasserwirtschaft. Osterr. Wasser u. Abfallwirtsch. Diese Ausgabe.

Syme WJ (1991) Dynamically Linked Two-DiModelling Program for Rivers, Estuaries \& Codelling Program for Rivers, Estuaries \& (100\% Research) Thesis, Dept of Civil En neering. The University of Queensland, May 1991 . The University of Queensland, May Van Riesen S (1975) Uferfiltratverminderung durch Selbstdichtung ä Karlsruhe. Dissertation, Universität Karlsruhe. rence Manual, WBM Oceanics Australia. 